\title{
Impact of vaccine delays at the 2, 4, 6 and 12 month visits on incomplete vaccination status by 24 months of age in Quebec, Canada
}

Marilou Kiely ${ }^{1,2^{*}}$ (D) Nicole Boulianne ${ }^{1}$, Denis Talbot ${ }^{2,3}$, Manale Ouakki ${ }^{1}$, Maryse Guay ${ }^{1,4,5}$, Monique Landry ${ }^{6}$, Chantal Sauvageau ${ }^{1,2,3}$ and Gaston De Serres ${ }^{1,2,3}$

\begin{abstract}
Background: Timeliness in the administration of recommended vaccines is often evaluated using vaccine delays and provides more information regarding the susceptibility of children to vaccine-preventable diseases compared with vaccine coverage at a given age. The importance of on-time administration of vaccines scheduled at the first visit is well documented, but data are scarce about the impact of vaccine delays at other visits on vaccination status by 24 months of age. Using vaccine delays for the first three doses of DTaP-containing vaccines and for the first dose of measles-containing vaccines as markers of timeliness at the 2, 4,6 and 12 month visits, we estimated the proportion of incomplete vaccination status by 24 months of age attributable to a vaccine delay at each of these visits.

Methods: We used the data from six cross-sectional coverage surveys conducted in the Province of Quebec from 2006 to 2016 which included 7183 children randomly selected from the universal health insurance database. A vaccine dose was considered delayed if received 30 days or more after the recommended age. The impact of new vaccine delays at each visit on incomplete vaccination status by 24 months of age was estimated with the attributable risk in the population.

Results: The proportion of children with vaccine delay was $5.4 \%$ at 2 months, $13.3 \%$ at 4 months, $23.1 \%$ at 6 months and $23.6 \%$ at 12 months. Overall, $72.5 \%$ of all 2-year-old children with an incomplete status by 24 months were attributable with a vaccine delay, of which $16.1 \%$ were attributable with a first vaccine delay at 2 months, $10.6 \%$ at 4 months, $14.0 \%$ at 6 months and $31.8 \%$ at 12 months.

Conclusions: While great emphasis has been put on vaccine delays at the first vaccination visit, the prevalence of vaccine delays was greater with later visits and most children with an incomplete vaccination status by 24 months had a vaccine delay occurring during these later visits. Interventions to improve timeliness should address vaccine delays at each visit and not only focus on the first visit.
\end{abstract}

Keywords: Delay, Timeliness, Vaccine coverage, Vaccination, Infant

\footnotetext{
* Correspondence: marilou.kiely@inspq.qc.ca

'Institut national de santé publique du Quebec, 2400 d'Estimauville Avenue,

Quebec G1E 7G9, Canada

${ }^{2}$ Department of Social and Preventive Medicine, Laval University, Quebec,

Canada

Full list of author information is available at the end of the article
}

(c) The Author(s). 2018 Open Access This article is distributed under the terms of the Creative Commons Attribution 4.0 International License (http://creativecommons.org/licenses/by/4.0/), which permits unrestricted use, distribution, and reproduction in any medium, provided you give appropriate credit to the original author(s) and the source, provide a link to the Creative Commons license, and indicate if changes were made. The Creative Commons Public Domain Dedication waiver (http://creativecommons.org/publicdomain/zero/1.0/) applies to the data made available in this article, unless otherwise stated. 


\section{Background}

Vaccine coverage is a common indicator used for the evaluation and monitoring of vaccination programs. It evaluates the proportion of individuals who have received all the recommended vaccines, regardless of the timeliness [1]. Timely vaccination is increasingly used to monitor vaccination programs. By assessing the interval between the recommended age and the age at which a dose was administered, this indicator provides a better estimate of the period during which children are protected [2-4]. Timely vaccination is often evaluated using vaccine delays for which several definitions exist. The definition most commonly used is a delay of 30 days or more after the recommended age for each dose [3-10]. A vaccine delay for a dose may impact on-time administration of subsequent doses and increase the child's risk of disease targeted by the vaccine $[11,12]$.

In Quebec (Canada), vaccines recommended to children are free of charge and are mostly administered by public health clinics. In 2005, a survey conducted by the Quebec Ministry of Health reported that vaccine delays to the childhood schedule were occurring in public health clinics of several regions of the province. These delays were attributed to a variety of factors including the introduction of new vaccine programs in the early 2000's [13, 14], missed opportunities due to providers reluctance to administer several injections at the same visit and barriers like difficulty to get a vaccination appointment. In response, monitoring of vaccine delays has been introduced in 2006 in public health clinics for the 2 -month and the 12-month visits. To improve the 2 -month visit timeliness, appointment periods with priority given to first visits were added and activities such as reminders and recalls were implemented. For children with vaccine delays, an accelerated vaccination schedule can be applied using the minimum intervals between the vaccine doses instead of the intervals recommended for routine vaccination.

Since 2006, vaccine coverage surveys have been conducted every 2 years in children aged 1 and 2 years. The 2006 survey found that only $17 \%$ of 24 -month old children had received all recommended vaccine doses with no delays. This survey as well as other studies had shown that vaccine delays of 30 days or more for vaccines scheduled at the first visit at 2 months of age were associated with vaccine delays at later visits or with an incomplete vaccination status by 24 months of age [9, 15-18]. However there are scarce data regarding the frequency of vaccine delays at other visits and their impact on vaccine coverage by 24 months of age. Using Quebec vaccine coverage surveys from 2006 to 2016, we estimated the proportion of children with vaccine delays at 2, 4, 6 and 12 months and the proportion of incomplete vaccination status by 24 months of age attributable to a vaccine delay at each of these visits. To identify more vulnerable populations, factors associated with vaccine delays at each vaccination visit from 2 to 12 months were assessed.

\section{Methods}

Study population and survey design

The present study is based on the data from six cross-sectional surveys conducted in Quebec in 2006, 2008, 2010, 2012, 2014 and 2016 [15, 19-23]. With the authorization of the Quebec Access to Information Commission, children in these studies were randomly selected from the Quebec Universal Health Insurance database which includes all children from the province. Each survey included a "1-year cohort" and a "2-year cohort" with children aged 15 to 17 months and 24 to 26 months respectively at the time of the survey. With surveys conducted every other year and cohorts defined by year of birth (e.g. in the survey conducted at the beginning of 2006, the 2-year cohort included children born in 2003 and those in the 1-year cohort were born in 2004), the six surveys assessed the immunization data in 12 different birth cohorts (born between 2003 and 2014). The surveys invited approximately 1000 children in each cohort (only 600 for 2006), a number deemed sufficient to obtain a precision of $\pm 3 \%$ in the vaccine coverage for each survey assuming a response rate of about $60 \%$ [24]. Were excluded children living in the two northern regions of the province.

Questionnaires were sent by mail and were filled out by parents or legal guardians. A postal reminder was sent to non-respondents 2 weeks and 4 weeks later. In the absence of response, parents were called directly 2 weeks after the last postal reminder and those with unknown phone number or not reached by phone received another questionnaire by mail. Respondents were invited to transcribe on the questionnaire the information available in their child's vaccination booklet (i.e. vaccine names, date of vaccination and vaccine providers). The questionnaire also collected information on the characteristics of the child, the mother and the parents. For children without vaccination booklets and those with information incomplete or inconsistent with the provincial vaccine schedule, vaccine providers were contacted to collect/validate the information. Only written information on doses from parents or vaccine providers were accepted. Each survey was approved by the Ethic Board Committee of the CHU de Quebec-Université Laval Hospital and written consent was obtained from all the participants.

\section{Vaccination schedule}

Since 2004, many new vaccines had been introduced in the Quebec's vaccination schedule (Table 1).The pneumococcal conjugate vaccine $(\mathrm{PCV})$ was introduced in December 


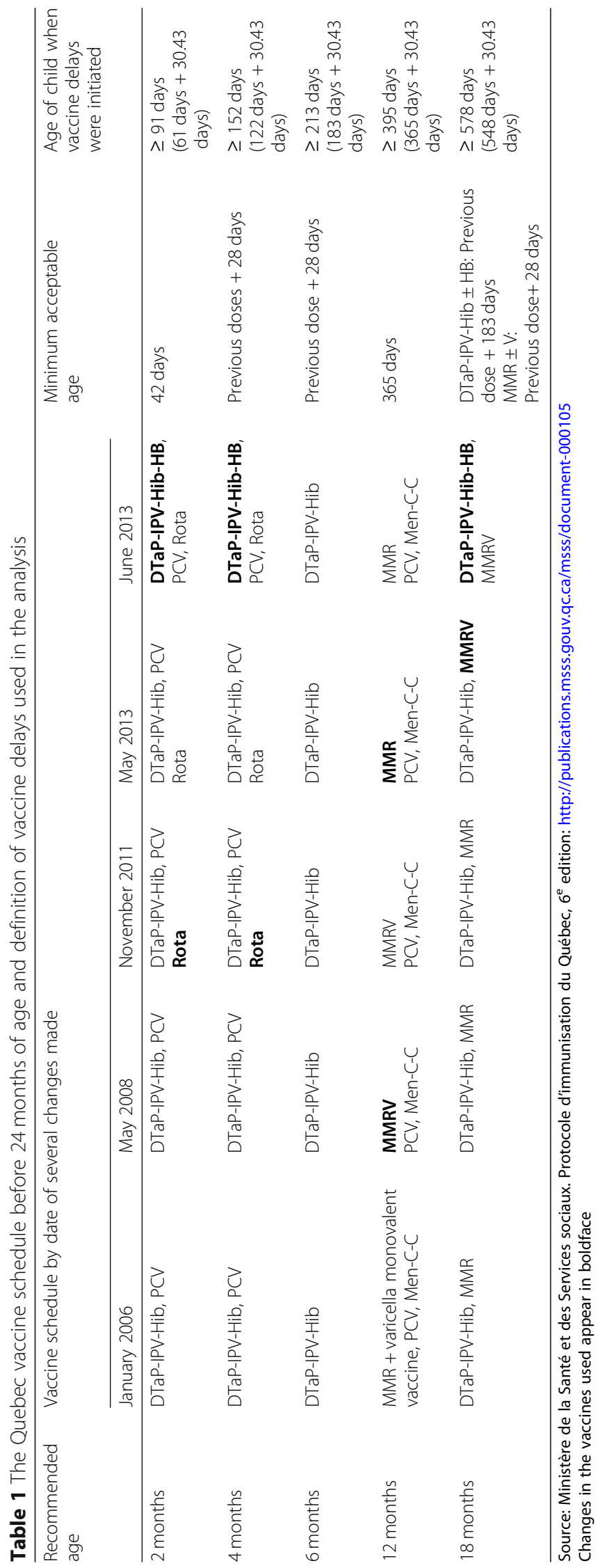


2004 with 3 doses scheduled at 2, 4 and 12 months. A single dose of monovalent varicella vaccine at 12 months of age was introduced in 2006 and replaced in 2008 by the combined measles, mumps, rubella and varicella vaccine (MMRV). Since November 2011, children are receiving two doses of rotavirus vaccine at 2 and 4 months of age. Finally, in June 2013, hepatitis B vaccine program was launched and the combined vaccine against diphtheria, acellular pertussis, tetanus, polio, Haemophilus influenzae type $\mathrm{b}$ and Hepatitis B (DTaP-IPV-Hib-HB) replaced the pentavalent vaccine DTaP-IPV-Hib in the schedule at 2, 4 and 18 months of age.

\section{Outcomes}

Birth and vaccination dates were used to compute age at vaccination in days. A dose was administered on-time if received within 30 days of the recommended age and was considered delayed after this period (Table 1). Vaccine delays were assessed for each of the first three doses of the $\mathrm{DTaP}$-containing vaccine (DTaP) recommended at 2,4 and 6 months of age and for the first dose of the measles-containing vaccine (Measles) recommended at 12 months. As most recommended vaccines are administered at the same visit, these vaccines have been used as markers of all vaccines administered at each visit. A vaccine delay at one dose is frequently followed by a vaccine delay at the following dose(s). A new vaccine delay was defined as a delay occurring among children whose previous dose(s) were on-time (e.g. Delay at 4 months among children with no delay at 2 months).

Given the changes in the Quebec vaccination schedule over the study period, the vaccination status was defined for antigens common to all surveys (DTaP-IPV-Hib, Men $\mathrm{C}-\mathrm{C}$ and measles-mumps-rubella). A complete vaccination status by 24 months of age was defined as having received 4 doses of DTaP-IPV-Hib, 1 dose of Men-C-C vaccine and 2 doses of MMR vaccine before 2 years of age. Otherwise, the vaccination status was incomplete.

\section{Statistical analysis}

All analyses were performed with Statistical Analysis System (SAS Institute Inc. Carry, NC, version 9.4). Proportions were compared using Chi-square test or Chi-square test for trend when appropriate. Missed opportunities at 2 and 12-month visits occurred when a vaccine-eligible child did not receive all recommended vaccines at the same vaccination date [25]. Other independent variables included data regarding the child, the family and the vaccine provider. The data on vaccine provider at the 2 month visit were used for the analysis of vaccine delays at 2, 4 and 6 months and the data on vaccine provider for all vaccination visits from 2 to 12 months were used for the analysis of vaccine delays at 12 months.
As vaccine delays occurred before 15 months of age, the analyses on factors associated with new vaccine delays included all participants to maximize the statistical power. In contrast, as children in the "1-year cohort" had not yet reached 2 years of age, the impact of new vaccine delays at 2, 4, 6 or 12 months on vaccination status by 24 months of age was estimated only with children in the "2-year cohort".

The impact of new vaccine delays at 2, 4, 6 and 12 month on vaccination status by 24 months of age was estimated with a robust Poisson multivariable regression. This alternative to logistic regression for binary outcomes directly estimates relative risk without risking convergence issues associated with log-binomial regressions [26-28]. The SAS GENMOD procedure was used with a log link and a robust estimator of the standard errors. All potential factors associated to incomplete vaccine coverage and to vaccine delay were included in the model without any selection procedure [29]. Risk-ratio modification by the survey year was assessed with an interaction term between vaccine delay and survey year in the model. A model based standardization approach was used to estimate adjusted risk difference (RD) in incomplete vaccination status by 24 months of age between children with and without vaccine delays [30]. Briefly, using predicted probabilities of the model, the standardized risk of outcome considering all children exposed (with vaccine delays) was estimated. The standardized risk considering all children unexposed (without vaccine delays) was also estimated similarly. The standardized $\mathrm{RD}$ was obtained as the difference between these two quantities. Adjusted attributable risks in the population (ARp) were estimated for delays at each dose also using a model based standardization approach. That is, ARp were estimated as one minus the ratio of the predicted risk of outcome considering all children unexposed over the observed risk of outcome [31]. Confidence intervals for $\mathrm{RD}$ and $\mathrm{ARp}$ were obtained through the percentile method by performing nonparametric bootstrap with 1000 samples [32, 33].

Factors associated with new vaccine delays were assessed for each dose with the same SAS procedure used to estimate the effect of vaccine delays on vaccination status by 24 months of age. A backward procedure with a $p$ value $<0.05$ was used for the selection criteria. The year of survey was kept in multivariable models to ensure face validity.

\section{Results}

Characteristics of participants, vaccine delays and vaccine coverage

Of the 11,200 children invited to participate since 2006, the participation rate was $70 \%(844 / 1200)$ in $2006,64 \%$ 
$(1282 / 2000)$ in $2008,62 \%(1233 / 2000)$ in $2010,73 \%$ $(1459 / 2000)$ in $2012,69 \%(1384 / 2000)$ in 2014 and $65 \%$ $(1295 / 2000)$ in 2016. Children born outside Quebec were excluded from the analysis because they were exposed to a different vaccine schedule $(n=140$ for the 1 -year cohorts and $n=174$ for the 2 -year cohorts). This left a total of 3675 children in the 1-year cohorts and 3508 children in the 2 -year cohorts (95\% of participants for both cohorts) for the analysis.

Forty-four percent of participant children were the first child in the family, $76.9 \%$ attending daycare and the majority were vaccinated in public health clinics $(66.7 \%)$. Most parents lived with a partner (91.9\%) and most mothers have completed a college or university degree (70.5\%). Missed opportunities occurred in $3.1 \%$ of children at the 2-month visit for which two injections were to be administered and in $14.8 \%$ at the 12 -month visit where three injections were recommended (from $31.1 \%$ in 2006 to $7.0 \%$ in 2016). All characteristics of children participants are presented in Additional file 1.

The proportion of children vaccinated by age in days for each dose is presented in Fig. 1. At 2 months, a greater proportion of children received their DTaP1 close to the recommended age compared to subsequent visits. At 6 months, $14.1 \%$ of children received DTaP3 after 227 days.
The proportion of new vaccine delays increased from 5.4\% at 2 months to $14.3 \%$ at 12 months (Fig. 2). Among children who experienced vaccine delays at 2 months $(n=$ 386), $77.2 \%$ had vaccine delays at other visits (298/386) (Fig. 3) and $33.7 \%$ had vaccine delays at each of the next three visits. At 4 months there were 2.4 times more vaccine delays $(n=941)$ than at 2 months of which $66.6 \%$ (627) were new vaccine delays. Among these new vaccine delays, $84.1 \%$ experienced vaccine delays at subsequent visits $(527 / 627)$. At 6 months, there were 4.2 times (1617) more vaccine delays than at the 2 month visit and $49.9 \%$ (807) were new vaccine delays. At 12 months, the number of vaccine delays was similar to that at 6 months (1624) and $60.7 \%$ (986) were new vaccine delays (Fig. 2 and Table 2). In children with a vaccine delay for DTaP doses, instead of using the minimum interval recommended for accelerated schedule to prevent additional vaccine delays, the majority received their next dose with 2-month intervals as recommended for routine schedule (see Additional file 2).

The characteristics of participants according to vaccine delays at 2, 4, 6 and 12 months of age are presented in Table 2. The proportion of children with a vaccine delay increased with age, from $5.4 \%$ at the 2 -month visit to $23.1 \%$ at the 6 -month visit and $23.6 \%$ at the 12 -month

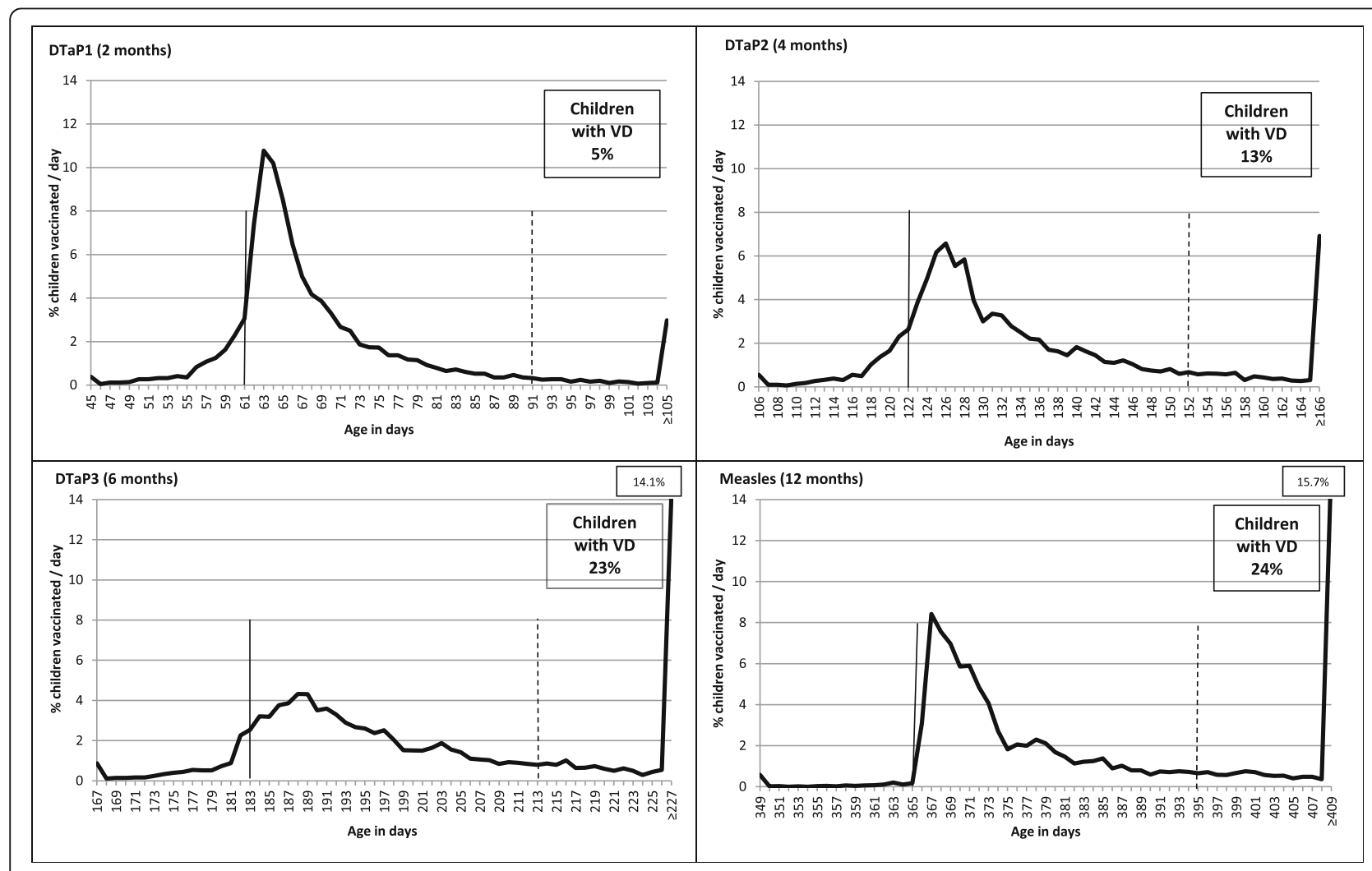

Fig. 1 Proportion of children vaccinated according to age in days at 2-4-6 and 12 months, 2006-2016* *Both cohorts included. The vertical reference lines indicate recommended age at vaccination for each visit and dotted lines indicate age when a dose becomes delayed 


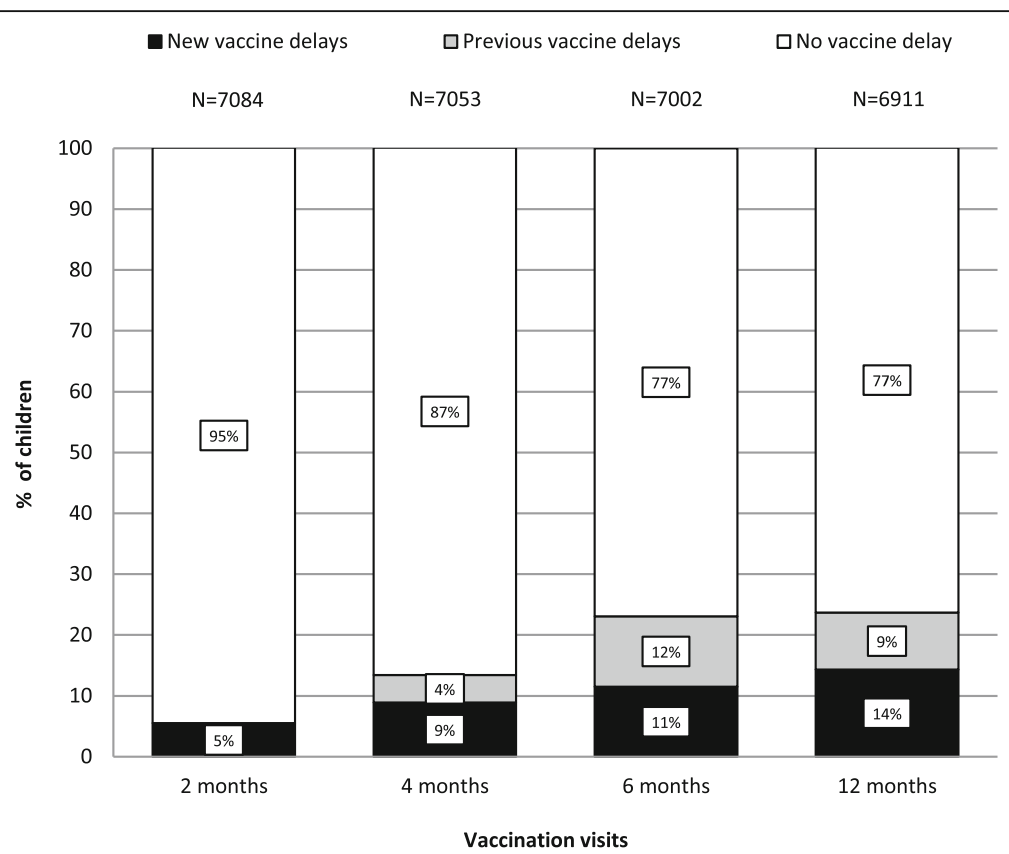

Fig. 2 Proportion of children with and without vaccine delays at 2-4-6 and 12 month visits, 2006-2016*. *Both cohorts included. DTaP1 is recommended at 2 months, DTaP2 at 4 months, DTaP3 at 6 months and the first measles-containing vaccine at 12 months

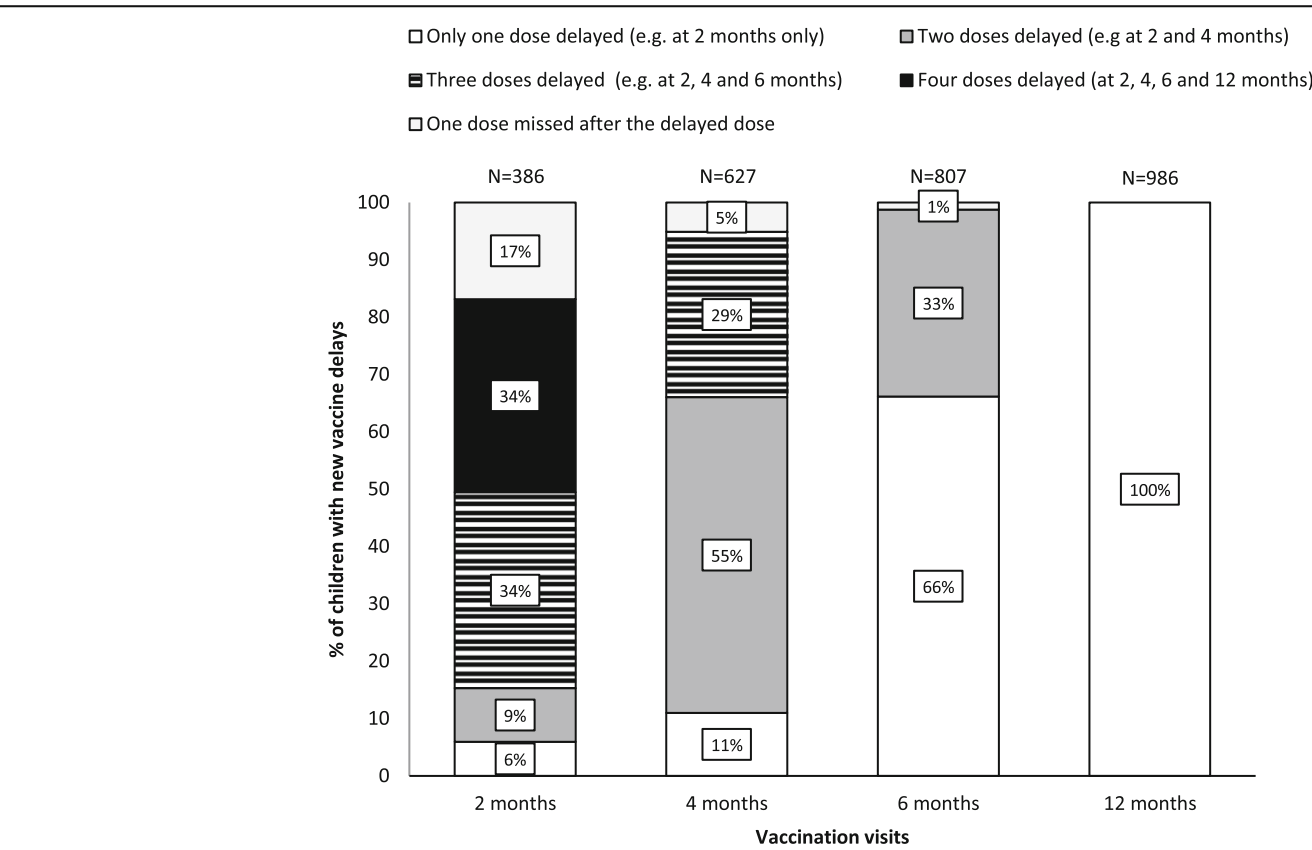

Fig. 3 Distribution of total vaccine delays by visit with first (new) vaccine delay, 2006-2016*. *Both cohorts included. DTaP1 was recommended at 2 months, DTaP2 at 4 months, DTaP3 at 6 months and the first measles-containing vaccine at 12 months. Categories of vaccine delays were mutually exclusive. The maximum number of delayed doses was 4 for a first (new) delay at 2 months, 3 at 4 months, 2 at 6 months and 1 at 12 months 


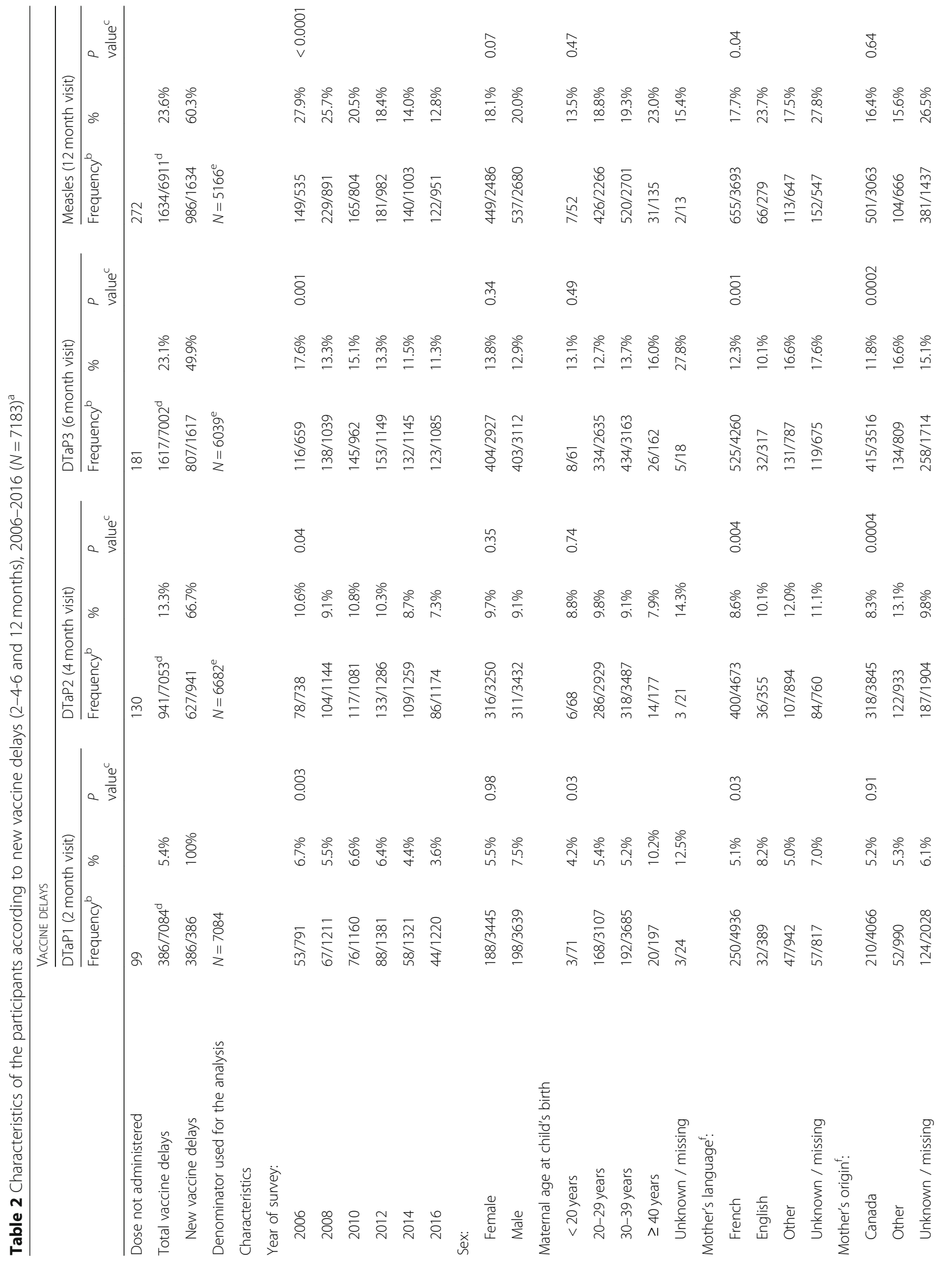




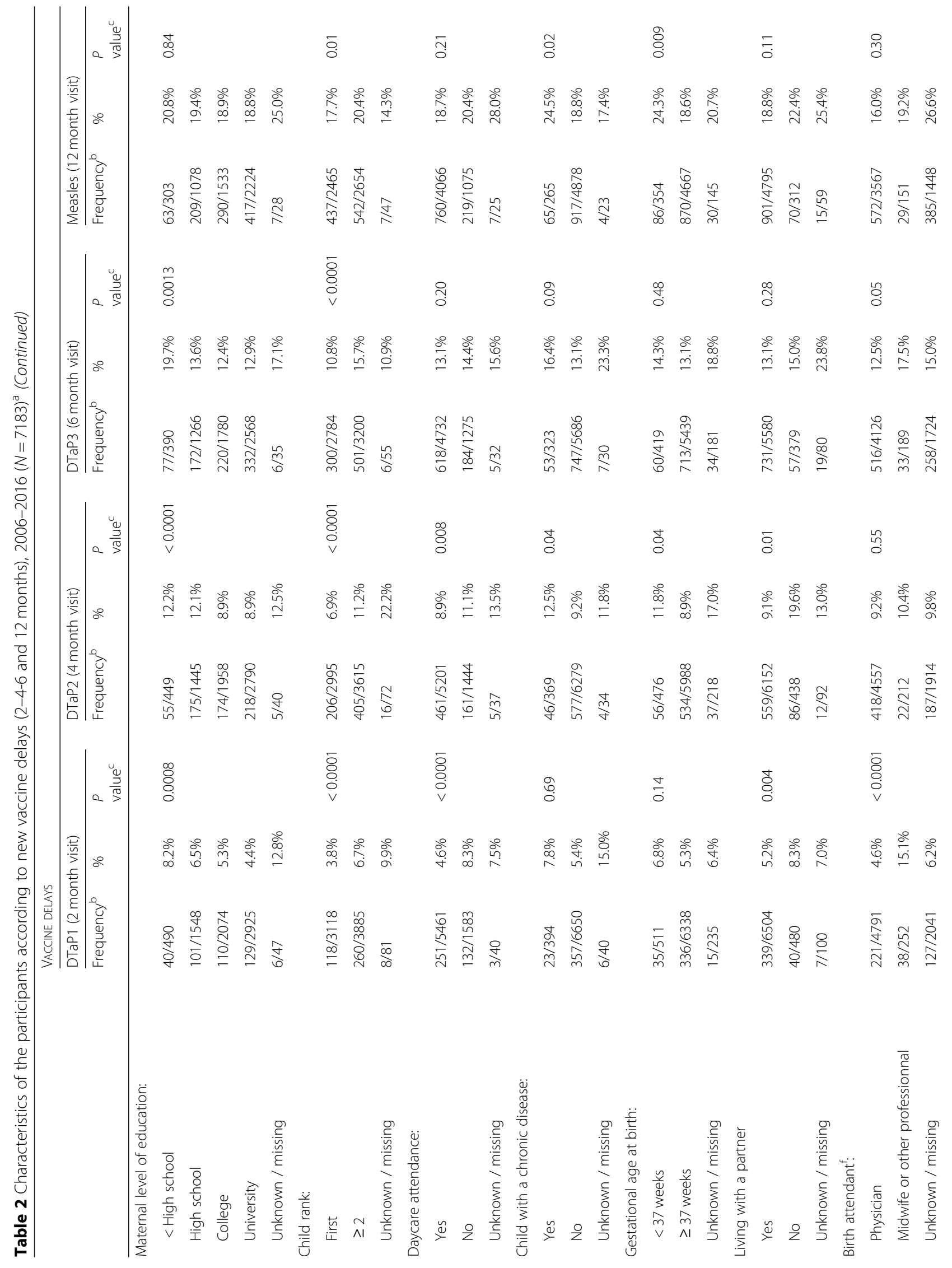


Kiely et al. BMC Public Health $\quad$ (2018) 18:1364

Page 9 of 15

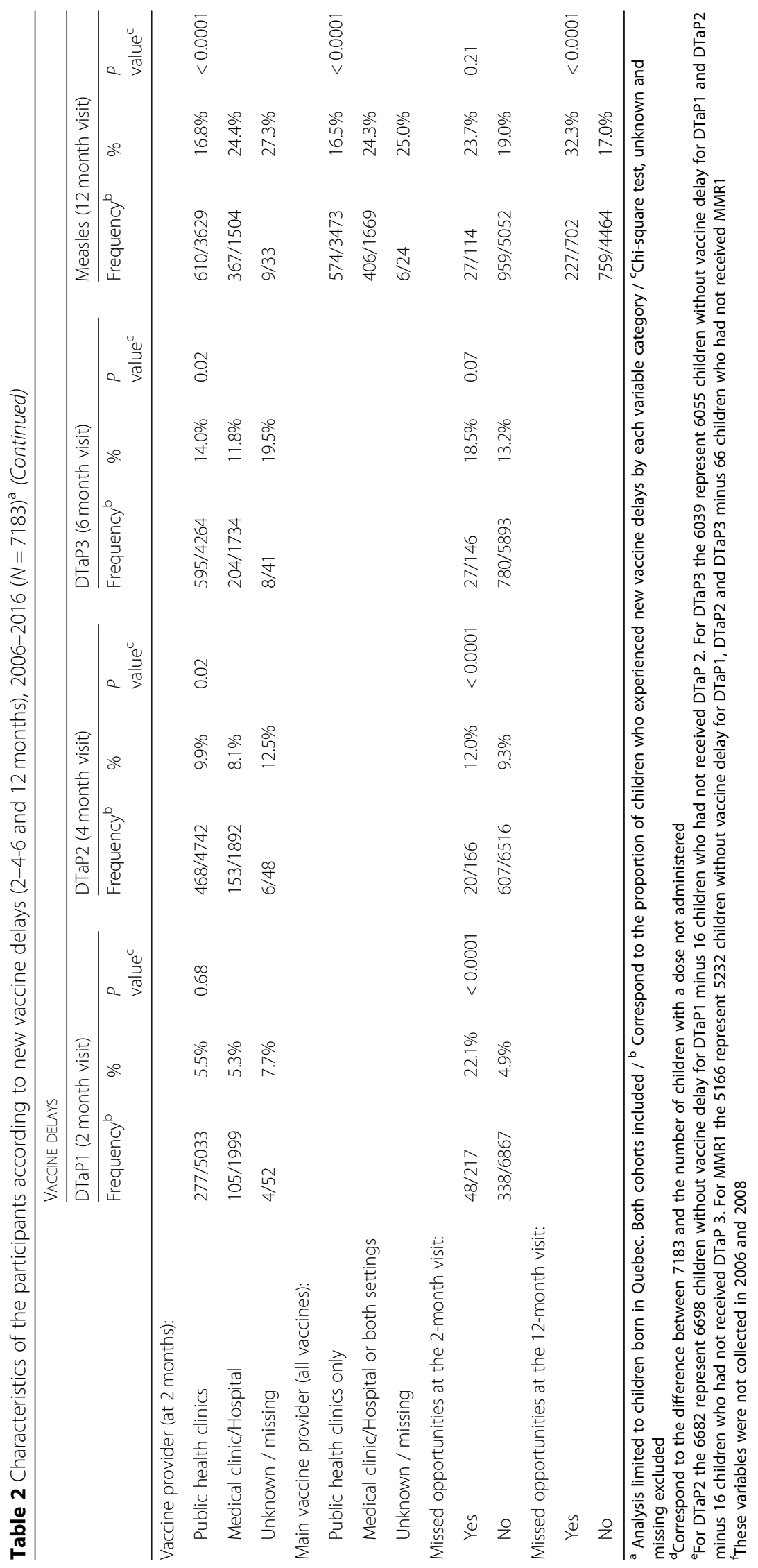


visit. Among children with a vaccine delay, the interval between 30 days after the recommended age and the time they received their dose was a median of 17 days for DTaP1 at 2 months, 10 days for DTaP2 at 4 months, 13 days for DTaP3 at 6 months and 21 days for measles at 12 months. Overall, $39 \%$ of children experienced at least one vaccine delay at one of the four visits, decreasing from $50 \%$ in 2006 to $30 \%$ in 2016. In univariate analysis, all characteristics except the sex were associated with new vaccine delays for at least one of the visits (Table 2). Between 2006 and 2016, for children immunized in public health clinics, the proportion with new vaccine delays decreased from 9.1 to $3.6 \%$ at the first visit, from 13.7 to $6.5 \%$ at the 4 month visit, from 20.3 to $11.0 \%$ at the 6 month visit and from 31.0 to $11.2 \%$ at the 12 month visit. In contrast, no significant differences were observed for children vaccinated in medical clinic/hospital for the 4 and 6 month visits (see Additional file 3).

\section{Impact of new vaccine delays on vaccination status by 24 months of age}

Among children with and without a vaccine delay for the DTaP1 at 2 months, 46.4 and $11.1 \%$ respectively had an incomplete vaccination status by 24 months for an unadjusted risk difference of $35.3 \%$ and a $27.7 \%$ adjusted risk difference (RD) (Table 3). The adjusted risk difference for incomplete vaccination status by 24 months was $13.8,10.5$ and $8.8 \%$ for new vaccine delays at 4,6 and 12 months respectively. The relative risk of incomplete vaccination status by 24 months of age between children with and without a vaccine delay varied between 2.3 and 3.6 with overlapping confidence intervals. As computed with predicted probabilities from the multivariable models, $72.5 \%$ of 2-year children with an incomplete status by 24 months were attributable to a vaccine delay: $16.1 \%$ were attributable to a vaccine delay that first occurred at 2 months, $10.6 \%$ at 4 months, $14.0 \%$ at 6 months and $31.8 \%$ at 12 months. There was no risk-ratio heterogeneity for the survey year.

\section{Factors associated with new vaccine delays}

Compared to the firstborn, other children were more likely to have a new vaccine delay at 2, 4, 6 and 12 months (Table 4). Missed opportunity at the 2-month visit was the most important factor associated with vaccine delays for DTaP1 (RR 4.28 (IC 3.18; 5.77)). At 2 months, children who were not attending daycare (versus attending daycare) and children whose parent who completed the survey was living without a partner (versus with a partner) were more likely to experience delays. Vaccination in medical clinic/hospital (versus in public health clinics) at the 2-month visit was associated with lower risk of vaccine delay for DTaP2 and DTaP3. The risk of vaccine delays for DTaP1 and DTaP2 decreased with higher maternal education. Missed opportunity at the 12-month visit was the most important factor associated with vaccine delays for measles at 12 months. Gestational age at birth lower than 37 weeks (vs $\geq 37$ weeks) was also associated with vaccine delays at 12 months. Finally, in contrast to results observed for DTaP2 and DTaP3, children vaccinated in medical clinic/hospital or in both settings for the 12-month visit were more likely to experience measles vaccine delays at 12 months compared with children vaccinated in public health clinics.

\section{Discussion}

While the literature on vaccine coverage put an emphasis on vaccine delays at 2 months, this study shows that the proportion of vaccine delays was greater at later visits increasing from $5.4 \%$ at 2 months, to $13.3 \%$ at 4 months, to $23.1 \%$ at 6 months and to $23.6 \%$ at 12 months. Vaccine delay at one visit had an impact on the timing and administration of subsequent doses as most children with vaccine delay for a DTaP dose received their subsequent dose with a 2-month interval rather than the shortened interval recommended in the accelerated schedule. A vaccine delay decreased the probability of having received all recommended vaccines by 24 months of age and $72.5 \%$ of incomplete status by 24 months of age were attributed to a new vaccine delay at 2, 4, 6 or 12 months $(16.1 \%$ at 2 months, $10.6 \%$ at 4 months, $14.0 \%$ at 6 months and $31.8 \%$ at 12 months).

This study found that delays at the first visit did contribute to incomplete vaccination status by 24 months of age and that more than $75 \%$ of children with a vaccine delay at 2-months also had vaccine delays at later visits including a third with vaccine delays at all next three visits. Association between vaccine delays at the first vaccination visit and later vaccine delays or incomplete vaccination status by 24 months of age has been widely reported [9, 16-18]. While other studies also identified that vaccine delays increased at subsequent vaccine visits $[9,11,34,35]$, they did not assess their impact on incomplete vaccination status by 24 months, the additional step this study provides.

The interventions deployed in public health clinics in Quebec since 2006 to improve timeliness at the 2 and 12 month visits seem to have successfully reduced vaccine delays at all visits. While only $17 \%$ of 2 -year-old children had received all recommended doses with no delay in 2006, this proportion had increased to $50 \%$ in 2016 [15, 23]. In a cohort of 361,901 children born from 2004 through 2012 in United States, 55\% of children had been fully vaccinated with no delay by 24 months of age [36]. Reminders or recall interventions sent by vaccine 


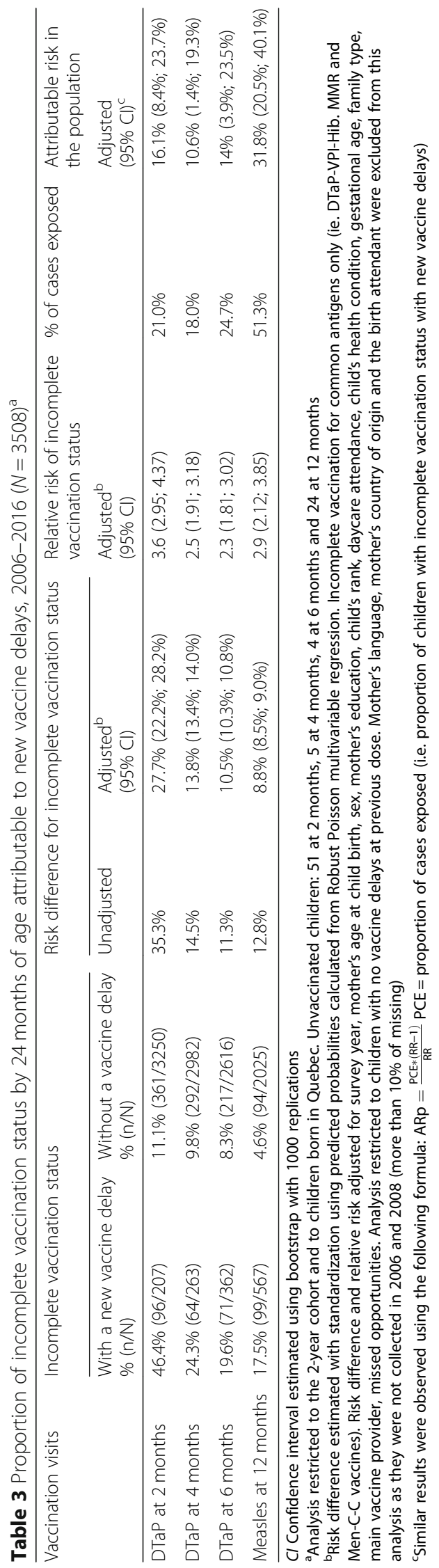




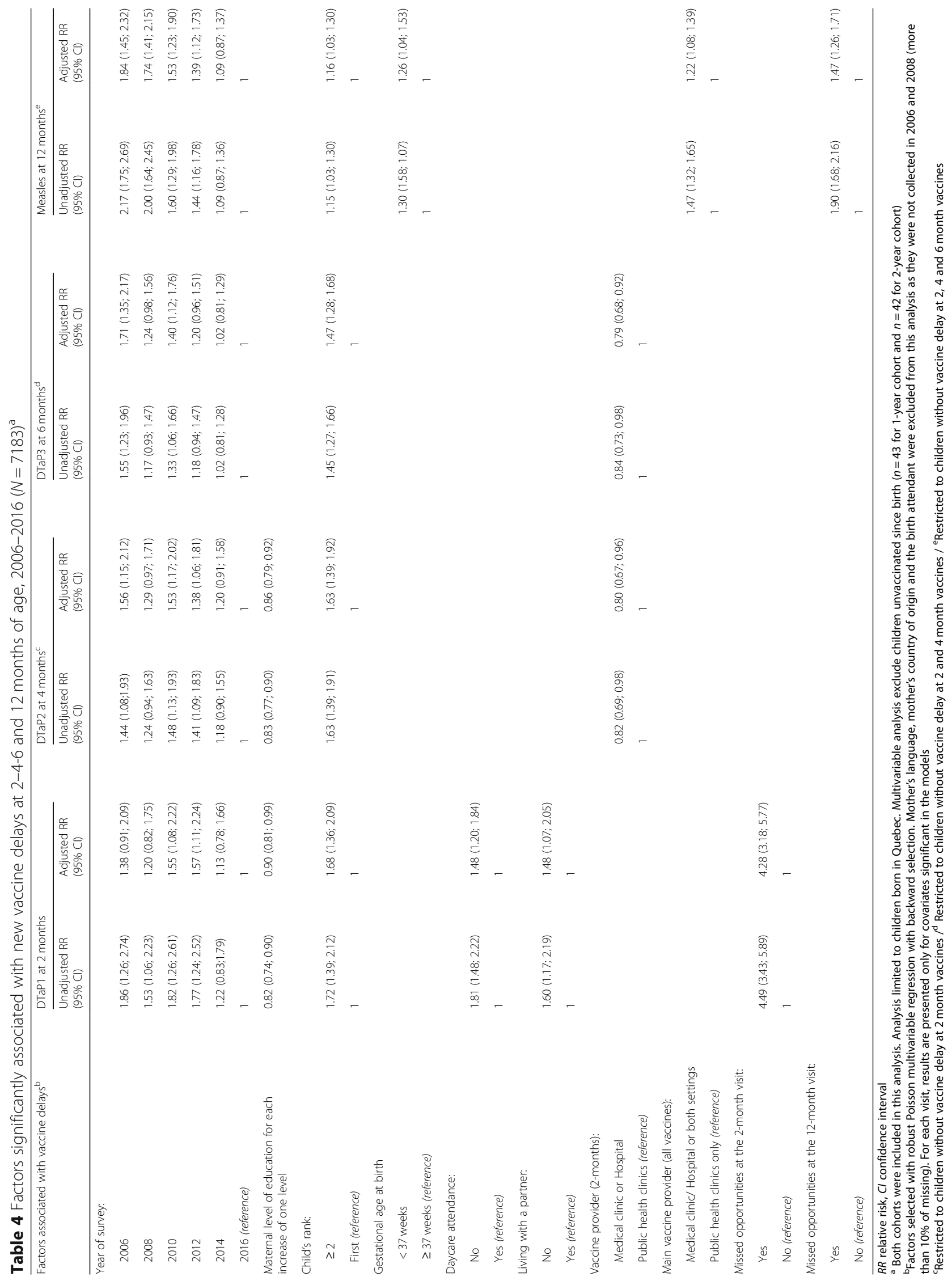


providers are effective to increase the likelihood of being vaccinated and to reduce the number of underimmunized days [37, 38]. In the current study, for children vaccinated in public health clinics, the proportion with new vaccine delays decreased from 2006 to 2016 for each vaccination visits: a decrease of $5.5 \%$ at 2 months, $7.2 \%$ at 4 months, $9.3 \%$ at 6 months and $19.8 \%$ at 12 months. The risk of vaccine delays for the 12 month visit was 1.22 times higher for children vaccinated in medical clinic/hospital versus those vaccinated in public health clinics. In contrast, in medical clinic/hospital where no intervention was done, no major changes have been observed in the proportion of new vaccine delays from 2006 to 2016. In public health clinics, efforts to reduced vaccine delays were more important for the 2 and 12 month visits and the proportions of children with vaccine delays at 4 and 6 months remained higher in this setting compared with medical clinic/hospital for each survey year except in 2016, resulting in a higher risk of vaccine delays.

In the present study, children who are not the firstborn and those with missed opportunities at the 2 -month and the 12-month visits were more likely to had vaccine delays. These two factors have been frequently associated with vaccine delays in other similar studies $[6,10,34,39]$. A high number of children in the household may impact the accessibility to healthcare settings, including vaccination services. In addition, children with older siblings are possibly more exposed to minor illness, resulting in missed opportunities and vaccine delays [40-42]. We observed that children of single parent (versus living with a partner) had a greater risk for vaccine delays at 2-months and these results might be associated with constraints to access vaccination services [6, 34]. In the current study, the risk of vaccine delays for DTaP1 and DTaP2 decreases with higher maternal education. The literature is inconsistent on this issue; some authors found an association similar to ours while others observed a higher risk of delays with an increasing education level $[6,10]$. A literature review found that higher maternal level of education was associated with vaccine hesitancy which is itself associated with vaccine delays [43]. This subject may require future studies.

This study has some limitations. The response rate to vaccine coverage surveys varied from 61 to $73 \%$ depending upon the year and cohort. Participants may have had more positive behaviours regarding vaccination than non-participants, resulting in less vaccine delays and higher vaccine coverage. Validation of the information on immunization from questionnaires was restricted to children whose information was not consistent with the provincial vaccine schedule. We cannot rule out that this practice has led to an overestimation of vaccine coverage, but the impact on our results might be minimal as vaccination card compared to medical chart usually has a good positive predictive value $[44,45]$. As only written data were accepted in this study, underestimation of vaccine coverage cannot be ruled out but is unlikely as many sources were consulted to obtain vaccine information. As markers of timeliness, vaccine delays were estimated only for DTaP and measles containing vaccines despite other vaccines being administered at 2, 4, 6 and 12 months. While most recommended vaccines are administered at the same visit, the burden associated with vaccine delays presented in this analysis has probably been underestimated.

The current study estimated the risk of incomplete vaccination status by 24 months attributable to vaccine delays at different ages an information that can be useful to decide or prioritize public health interventions. However, the interpretation of $A R p$ as the fraction of the outcome that could be eliminated if exposure could be totally removed from the population is valid only under certain conditions [46]. First, exposure has to be causal rather than merely associated with the disease. By estimating counterfactual risk with model-based standardization, we attempted to estimate the average causal effect of new vaccine delays at 2, 4, 6 or 12 months on vaccination status by 24 months. However, root causes of vaccine delays remained unknown and the elimination of vaccine delays may not result in an improvement of complete vaccination status by 24 months as large as estimated by ARp. Second, estimation of ARp has to be unbiased. While multivariable models included most of the determinants identified in the literature, as for any observational study, residual confounding cannot be ruled out. Finally, the elimination of exposure has to be without any effect on distribution of other risk factors. As other factors included in this analysis are mainly unmodifiable risk factors, it is unlikely that the elimination of vaccine delays has any effect on their distribution. As the attributable risk depends upon the prevalence of vaccine delays at various visits, which may vary between populations, this limits the generalizability of this result to other jurisdictions [46].

\section{Conclusion}

While great emphasis has been put on vaccine delays at the first vaccination visit, the prevalence of vaccine delays in this study was greater with later visits and most of incomplete vaccination status by 24 months of age was associated with vaccine delays occurring after the 2 month visit. Interventions to improve timeliness deployed in public health clinics in Quebec seem to have 
reduced vaccine delays at all visits from 2006 to 2016 . These interventions should also focus on other vaccine visits which contributed to the burden associated with vaccine delays and on more vulnerable groups identified in this study. Accelerated vaccination schedules recommended for children with vaccine delays may prevent further delays and reduce their impact.

\section{Additional files}

Additional file 1: Characteristics of children participants, 1-year and 2-year cohorts, 2006-2016 $(n=7183)$. (DOCX $20 \mathrm{~kb})$

Additional file 2: Intervals following the next vaccine dose for children with new vaccine delays at the DTaP1 and DTaP2. (DOCX $26 \mathrm{~kb}$ )

Additional file 3: New vaccine delays at 2, 4, 6 and 12 months by vaccine provider and survey year, 1-year and 2-year cohorts, 2006-2016. (DOCX $17 \mathrm{~kb})$

\section{Abbreviations}

ARp: Attributable risk in the population; Cl: Confidence interval; DTaP: Diphtheria, tetanus, acellular pertussis and polio containing vaccine; DTaP-IPV-Hib: Diphtheria, tetanus, acellular pertussis, polio virus and Haemophilus Influenzae type b vaccine; DTaP-IPV-Hib-HB: Diphtheria, tetanus, acellular pertussis, polio virus, Haemophilus Influenzae type b vaccine and Hepatitis B vaccine; Measles: Measles-containing vaccine; Men-C-C: Meningococcal C conjugate vaccine; MMR: Measles, mumps and rubella vaccine; MMRV: Measles, mumps, rubella and varicella vaccine; PCV: Pneumococcal conjugate vaccine; Rota: Rotavirus vaccine; RR: Risk ratio; VPD: Vaccine-preventable diseases

\section{Acknowledgements}

Not applicable.

\section{Funding}

MK has received a scholarship from the Fonds de recherche du Quebec-Santé (FRQS).

DT is a FRQS junior 1 Chercheur-Boursier.

Vaccine coverage surveys from 2006 were funded by the Ministère de la Santé et des Services sociaux du Québec. Funding sources had no involvement on the conduct of this research and on the redaction of this manuscript.

\section{Availability of data and materials}

The datasets used and analysed during this current study are available from the corresponding author on reasonable request.

\section{Authors' contributions}

NB, GDS and MK (since 2016) conceptualized the research and collected the data. MK performed analyses, interpreted the results and prepared the initial draft of the manuscript. GDS, DT and NB were supervisors of MK's PhD candidature and assisted in performing analyses and provided overall guidance to MK. MK, NB, GDS, MO, MG, ML and CS participated in the study design and methodology. All authors contributed significantly to towards drafting and editing the manuscript. All authors have read and approved the final version of the manuscript.

\section{Ethics approval and consent to participate}

Each survey from 2006 to 2016 was approved by the Ethic Board Committee of the CHU de Quebec-University Laval Hospital and written consent was obtained from all the participants.

\section{Consent for publication}

Not applicable.

\section{Competing interests}

Gaston De Serres received research grand for investigator-initiated studies from Pfizer and GlaxoSmithKline (GSK) and was reimbursed expenses to travel to an ad hoc advisory board meeting by GSK but received no honoraria. He has equally provided paid expert testimony in a grievance against a vaccine-or-mask healthcare worker influenza vaccination policy for the Ontario Nurse Association. Other authors have no conflicts of interest to declare.

\section{Publisher's Note}

Springer Nature remains neutral with regard to jurisdictional claims in published maps and institutional affiliations.

\section{Author details}

${ }^{1}$ Institut national de santé publique du Quebec, 2400 d'Estimauville Avenue, Quebec G1E 7G9, Canada. 'Department of Social and Preventive Medicine, Laval University, Quebec, Canada. ${ }^{3} \mathrm{CHU}$ de Québec-Université Laval, Quebec, Canada. ${ }^{4}$ Department of Community Health Sciences, Sherbrooke University, Quebec, Canada. ${ }^{5}$ Centre intégré de Santé et Services Sociaux de la Montérégie-Centre, Quebec, Canada. ${ }^{6}$ Ministère de la Santé et des Services Sociaux, Quebec, Canada.

Received: 23 April 2018 Accepted: 20 November 2018

Published online: 11 December 2018

References

1. Goldstein ND, Newbern EC, Evans AA, Drezner K, Welles SL. Choice of measures of vaccination and estimates of risk of pediatric pertussis. Vaccine. 2015;33(32):3970-5.

2. Dannetun E, Tegnell A, Hermansson G, Törner A, Giesecke J. Timeliness of MMR vaccination--influence on vaccination coverage. Vaccine. 2004; 22(31-32):4228-32.

3. Akmatov MK, Kretzschmar M, Kramer A, Mikolajczyk RT. Timeliness of vaccination and its effects on fraction of vaccinated population. Vaccine. 2008;26:3805-11.

4. Luman ET, Barker LE, Shaw KM, McCauley MM, Buehler JW, Pickering LK. Timeliness of childhood vaccinations in the United States days Undervaccinated and number of vaccines delayed. JAMA. 2005;293(10): 1204-11.

5. Bolton P, Hussain A, Hadpawat A, Holt E, Hughart N, Guyer B. Deficiencies in current childhood immunization indicators. Public Health Rep Wash DC 1974. 1998;113(6):527-32.

6. Dombkowski KJ, Lantz PM, Freed GL. Risk factors for delay in ageappropriate vaccination. Public Health Rep Wash DC 1974. 2004; 119(2):144-55.

7. Dietz V, Zell E, Eddins D, Bernier R, Orenstein W. Vaccination coverage in the USA. Lancet Lond Engl. 1994;344(8934):1439-40.

8. Hull BP, McIntyre PB. Timeliness of childhood immunisation in Australia. Vaccine. 2006;24(20):4403-8.

9. Strine TW, Luman ET, Okoro CA, McCauley MM, Barker LE. Predictors of age-appropriate receipt of DTaP dose 4. Am J Prev Med. 2003; 25(1):45-9.

10. Dayan GH, Shaw KM, Baughman AL, Orellana LC, Forlenza R, Ellis A, et al. Assessment of delay in age-appropriate vaccination using survival analysis. Am J Epidemiol. 2006;163(6):561-70.

11. Zhao Z, Smith PJ, Hill HA. Missed opportunities for simultaneous administration of the fourth dose of DTaP among children in the United States. Vaccine. 2017;35:3191-5.

12. Huang $\mathrm{W}-\mathrm{T}$, Lin $\mathrm{H}-\mathrm{C}$, Yang $\mathrm{C}-\mathrm{H}$. Undervaccination with diphtheria, tetanus, and pertussis vaccine: national trends and association with pertussis risk in young children. Hum Vaccines Immunother. 2017;13(4):757-61.

13. Public Health Agency of Canada. Canadian immunization guide. Available from: https://www.canada.ca/en/public-health/services/canadianimmunization-guide.html. [cited 6 Mar 2017].

14. Ministère de la Santé et des Services sociaux. Protocole d'immunisation du Québec, 6e édition. Available from: http://publications.msss.gouv.qc.ca/ msss/document-000105. [cited 20 Mar 2017]

15. Boulianne N, Audet D, Ouakki M, Guay M, Duval B, De Serres G. Enquête sur la couverture vaccinale des enfants québécois en 2006. Québec: Institut national de santé publique du Québec; 2007.

16. Fiks AG, Alessandrini EA, Luberti AA, Ostapenko S, Zhang X, Silber JH. Identifying factors predicting immunization delay for children followed in an urban primary care network using an electronic health record. Pediatrics. 2006:118(6):e1680-6.

17. Hanna JN, Wakefield JE, Doolan CJ, Messner JL. Childhood immunisation: factors associated with failure to complete the recommended schedule by two years of age. Aust J Public Health. 1994;18(1):15-21. 
18. Guerra FA. Delays in immunization have potentially serious health consequences. Paediatr Drugs. 2007;9(3):143-8.

19. Boulianne $N$, Bradet $R$, Audet $D$, Deceuninck $G$. Enquête sur la couverture vaccinale des enfants de 1 an et 2 ans au Québec en 2008. Québec: Institut national de santé publique du Québec; 2009.

20. Boulianne N, Bradet R, Audet D, Ouakki M, Guay M, De Serres G. Enquête sur la couverture vaccinale des enfants de 1 an et 2 ans au Québec en 2010. Québec: Institut national de santé publique du Québec; 2011.

21. Boulianne N, Bradet R, Audet D, Ouakki M, De Serres G, Guay M. Enquête sur la couverture vaccinale des enfants de 1 an et 2 ans au Québec en 2012. Québec: Institut national de santé publique du Québec; 2013.

22. Boulianne N, Audet D, Ouakki M, Dubé E, De Serres G, Guay M. Enquête sur la couverture vaccinale des enfants de 1 an et 2 ans au Québec en 2014. Québec: Institut national de santé publique du Québec; 2015.

23. Kiely M, Boulianne N, Ouakki M, Audet D, Gariépy MC, Guay M, De Serres G, Dubé E. Enquête sur la couverture vaccinale des enfants de 1 an et 2 ans au Québec en 2016. Québec: Institut national de santé publique du Québec; 2017.

24. Hanley JA, Moodie E. Sample size, precision and power calculations: a unified approach. J Biomet Biost. 2011;2(5):1000124.

25. Sabnis SS, Pomeranz AJ, Lye PS, Amateau MM. Do missed opportunities stay missed? A 6-month follow-up of missed vaccine opportunities in inner city Milwaukee children. Pediatrics. 1998;101(5):E5.

26. Skov T, Deddens J, Petersen MR, Endahl L. Prevalence proportion ratios: estimation and hypothesis testing. Int J Epidemiol. 1998;27(1):91-5.

27. Zou G. A modified poisson regression approach to prospective studies with binary data. Am J Epidemiol. 2004;159(7):702-6.

28. Barros AJD, Hirakata VN. Alternatives for logistic regression in cross-sectional studies: an empirical comparison of models that directly estimate the prevalence ratio. BMC Med Res Methodol. 2003;3:21.

29. VanderWele TJ, Shpitser I. A new criterion for confounder selection. Biometrics. 2011;67(4):1406-13.

30. Hernan MA, Robins JM. Causal inference. Boca Raton: Chapman \& Hall/ CRC, forthcoming; 2017

31. Gust DA, Strine TW, Maurice E, Smith P, Yusuf H, Wilkinson M, et al. Underimmunization among children: effects of vaccine safety concerns on immunization status. Pediatrics. 2004;114(1):e16-22.

32. Greenland S. Interval estimation by simulation as an alternative to and extension of confidence intervals. Int J Epidemiol. 2004;33(6):1389-97.

33. Kovar JG, JNK R, CFJ W. Bootstrap and other methods to measure errors in survey estimates. Can J Stat. 1988;16:25-45.

34. Lernout $\mathrm{T}$, Theeten $\mathrm{H}$, Hens $\mathrm{N}$, Braeckman $\mathrm{T}$, Roelants $\mathrm{M}$, Hoppenbrouwers $K$, et al. Timeliness of infant vaccination and factors related with delay in Flanders, Belgium. Vaccine. 2014;32(2):284-9.

35. Walton S, Cortina-Borja M, Dezateux C, Griffiths LU, Tingay K, Akbari A, et al. Measuring the timeliness of childhood vaccinations: using cohort data and routine health records to evaluate quality of immunisation services. Vaccine. 2017;35(51):7166-73.

36. Daley MF, Glanz JM, Newcomer SR, Jackson ML, Groom HC, Lugg MM, et al. Assessing misclassification of vaccination status: implications for studies of the safety of the childhood immunization schedule. Vaccine. 2017;35(15):1873-8.

37. Hambidge SJ, Phibbs SL, Chandramouli V, Fairclough D, Steiner JF. A stepped intervention increases well-child care and immunization rates in a disadvantaged population. Pediatrics. 2009;124(2):455-64

38. Jacobson Vann JC, Jacobson RM, Coyne-Beasley T, Asafu-Adjei JK, Szilagyi PG. Patient reminder and recall interventions to improve immunization rates. Cochrane Database Syst Rev. 2018;(1). Art. No.: CD003941.

39. Tozzi AE, Piga S, Corchia C, Di Lallo D, Carnielli V, Chiandotto V, et al. Timeliness of routine immunization in a population-based Italian cohort of very preterm infants: results of the ACTION follow-up project. Vaccine. 2014; 32(7):793-9.

40. Gust DA, Darling N, Kennedy A, Schwartz B. Parents with doubts about vaccines: which vaccines and reasons why. Pediatrics. 2008;122(4):718-25.

41. Luman ET, McCauley MM, Shefer A, Chu SY. Maternal characteristics associated with vaccination of young children. Pediatrics. 2003;111(5 Pt 2):1215-8.

42. Reading $R$, Surridge $H$, Adamson R. Infant immunization and family size. J Public Health Oxf Engl. 2004;26(4):369-71.
43. Larson HJ, Jarrett C, Eckersberger E, Smith DMD, Paterson P. Understanding vaccine hesitancy around vaccines and vaccination from a global perspective: a systematic review of published literature, 2007-2012. Vaccine. 2014:32(19):2150-9.

44. Goldstein KP, Kviz FJ, Daum RS. Accuracy of immunization histories provided by adults accompanying preschool children to a pediatric emergency department. JAMA. 1993;270(18):2190-4.

45. Miles M, Ryman TK, Dietz V, Zell E, Luman ET. Validity of vaccination cards and parental recall to estimate vaccination coverage: a systematic review of the literature. Vaccine. 2013;31(12):1560-8.

46. Benichou J, Palma M. Measures of impact. In: Handbook of epidemiology. Second. Germany: Wolfgang Ahrens \& Iris Pigeot; 2014. p. 2489
Ready to submit your research? Choose BMC and benefit from:

- fast, convenient online submission

- thorough peer review by experienced researchers in your field

- rapid publication on acceptance

- support for research data, including large and complex data types

- gold Open Access which fosters wider collaboration and increased citations

- maximum visibility for your research: over $100 \mathrm{M}$ website views per year

At BMC, research is always in progress.

Learn more biomedcentral.com/submissions 